\title{
Absceso de psoas brucelósico bilateral: presentación de un caso y revisión de la literatura
}

\author{
J. Romero Otero, V. Martínez Silva, M. Pamplona Casamayor, C. Capitán Manjón, \\ J. Piedra Lara, O. Leiva Galvis
}

Servicio de Urología. Hospital Universitario 12 de Octubre. Madrid.

Actas Urol Esp 2005; 29 (7): 704-707

\section{RESUMEN \\ ABSCESO DE PSOAS BRUCELÓSICO BILATERAL: PRESENTACIÓN DE UN CASO Y REVISIÓN DE LA LITERATURA}

La brucelosis es una zoonosis poco frecuente, aunque es endémica en España. Suele manifestarse como una enfermedad sistémica con presentación aguda o crónica, en ocasiones lo hace de manera localizada afectando a uno o varios aparatos.

La presentación focal más frecuente es la forma osteoarticular. Ésta se puede complicar en ocasiones con abscesos para-perivertebrales, la afectación del psoas unilateral es rara y en casos excepcionales existe extensión a ambos músculos bilateralmente.

Las manifestaciones clínicas de los abscesos de psoas(AP) suelen ser insidiosas, es infrecuente la aparición de la triada clásica de fiebre, dolor lumbar e impotencia funcional. Para el diagnóstico son necesarias las pruebas serológicas y medios de cultivo. El uso extendido de la Ecografia (ECO) y la Tomografía Axial Computerizada (TAC) ha mejorado el diagnóstico y tratamiento de las complicaciones. El tratamiento de elección es antibiótico y se basa en el uso de tetraciclinas y estreptomicina. En ocasiones se hace necesaria la punción percutánea o drenaje abierto de los abscesos.

Presentamos un caso de AP brucelósico bilateral y revisamos la bibliografía.

Palabras clave: Absceso. Psoas. Brucelosis. Espondilitis. Sacroileitis.

\section{ABSTRACT}

BILATERAL BRUCELLOSIC PSOAS ABSCESS: ONE CASE IS RELATED AND LITERATURE REVIEW

The Brucellosis is a zoonotic and unfrequent infection but it is endemic in Spain. It is a well documented cause of fever of unknown origin with varied and nonspecific symptoms. The onset of symptoms of brucellosis may be abrupt or insidious, developing over several days to weeks. Virtually any organ system can be involved with brucellosis and localization of the process may cause focal symptoms.

The most frequent focal presentation is the osteoarthicular. Some times it can complicates with paraperivertebral abscess. The unilateral psoas abscess(PA) is uncommon and the bilateral afection is exceptional.

The manifestations of PA usually are insidious, the classic tiad of: feber, lumbar pain and functional impotence is rare.

For the diagnosis the serologyc tests and cultures are necesary. The imagin tecniques like: Ultrasonography and Tomography have improved the diagnosis and treatment of this patologys complications.

The recomended therapy is the use of doxycicline and streptomicine. Some times the use of percutaneus drainage or open surgery is necesary.

Keywords: Abscess. Psoas. Brucellosic. Spondylitis. Sacroileitis. 
$\mathrm{L}^{\mathrm{a}}$ a afectación localizada más frecuente de la brucelosis es la osteoarticular. Se suele presentar en forma de sacroileitis o de espondilodiscitis. Estas entidades en raras ocasiones se complican con la aparición de un absceso. Presentamos el caso de un paciente diagnosticado de espondilodiscitis brucelósica complicado con absceso de psoas, el hecho de ser bilateral lo hace excepcional.

\section{CASO CLÍnICo}

Un varón de 42 años de edad, residente en Mérida (Extremadura), con antecedentes personales de diabetes mellitus tipo I y prostatitis crónica; consultó a las tres semanas de la ingesta de un producto lácteo no higienizado por fiebre y malestar general. Las pruebas complementarias mostraban un hemograma, bioquímica, pruebas de coagulación y radiología convencional sin alteraciones. Los hemocultivos en medios convencionales y de Ruiz Castañeda fueron negativos. El estudio serológico para Brucella resultó: Rosa de Bengala: positivo, sero-aglutinación: positiva (1/20) y antibrucela-coombs: positivo.

Se inició tratamiento con Rifampicina 900 mgr/dia más doxaciclina 100mgr/12h durante 6 semanas.

Transcurridos cinco meses sufre una recaída y presenta dolor lumbar más fiebre. A la exploración la región ilíaca izquierda está empastada junto con signo del psoas positivo. Se le realizó una tomografía axial computerizada (TAC) en la que se objetivó una espondilodiscitis L5-S1 con afectación de partes blandas circundantes y absceso de psoas bilateral de pequeño tamaño. Se decide reanudar tratamiento antibiótico previo al que se agrega ciprofloxacino 400mgr/12h. Transcurrido un mes, dada la mala respuesta al tratamiento se realiza drenaje transcutáneo de las colecciones del psoas.

Ante la mala evolución del paciente se practicó una nueva TAC en la que se podía observar la existencia de abscesos de psoas bilaterales de gran tamaño y afectación de tejido circundante junto con espondilodiscitis en L5-S1 (Figs. 1, 2 y 3). El paciente fue remitido a nuestro servicio donde fue intervenido por el servicio de urología y neurocirugía conjuntamente realizándose un drenaje de ambos músculos y la discectomía L5-S1.

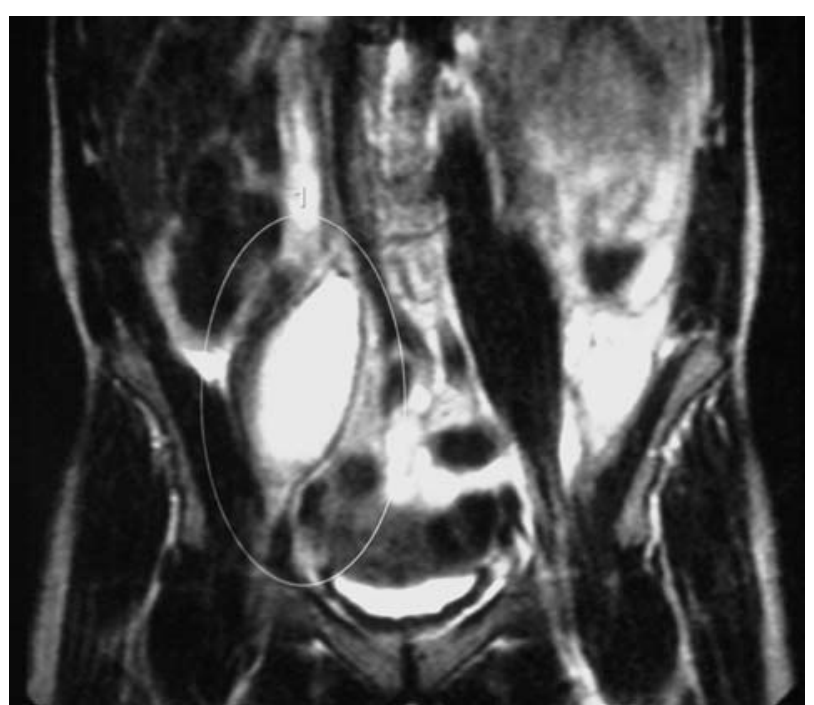

FIGURA 1. Absceso de psoas corte longitudinal.

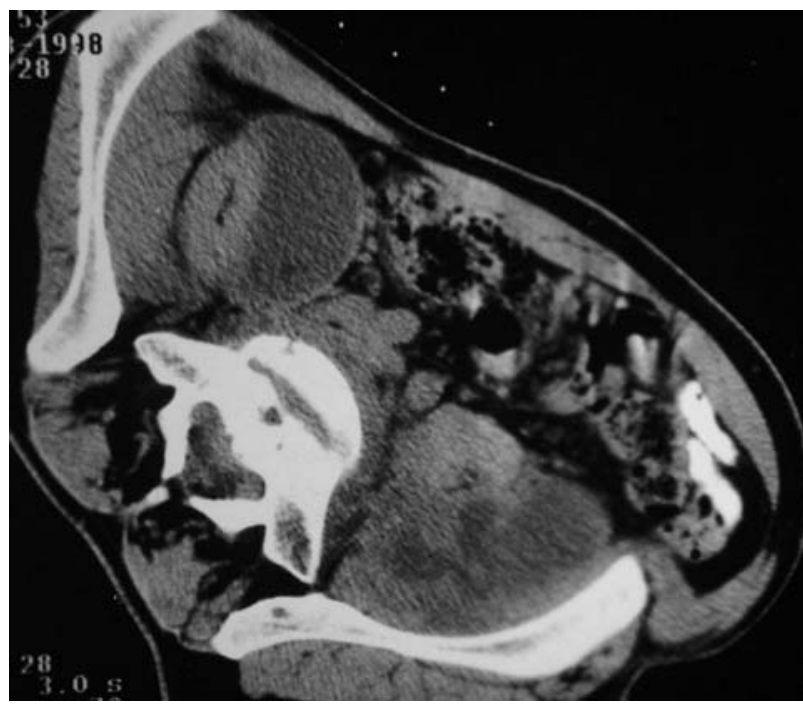

FIGURA 2. Absceso de psoas bilateral corte sagital.

El postoperatorio transcurrió sin incidencias por lo que se le dio el alta con tratamiento antibiótico doxiciclina 100mgr/12horas junto con septrim forte $1 / 12$ horas durante cuatro meses.

El paciente está asintomático con buen estado general hasta la última revisión tres años después de la cirugía.

\section{DISCUSIÓN}

El músculo psoas es grande con origen en las apófisis transversas de las vértebras D12 y lumbares, se fusiona con el ilíaco para ir a insertarse en el trocánter menor. Su función es la flexión de 


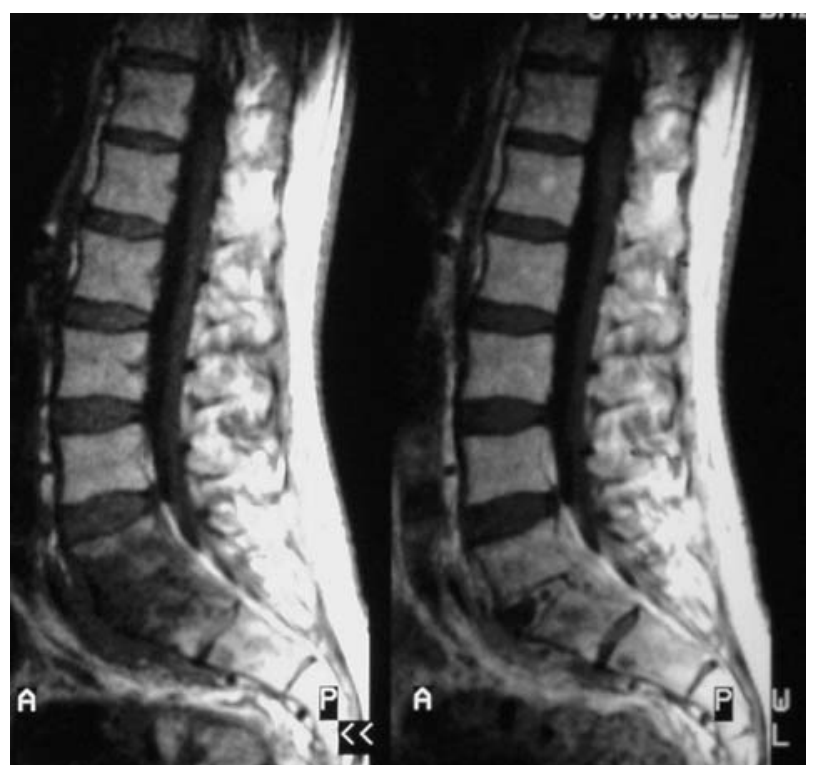

FIGURA 3. Espondilodiscitis L5-S1.

muslo y tronco principalmente ${ }^{1}$. Esto hace que tenga relaciones con multitud de órganos, por cuyas patologías se puede ver afectado; así en 1881 Mynter clasificó los abscesos de psoas (AP) en primarios cuando la infección está localizada exclusivamente en el músculo y secundarios si se extienden desde un foco adyacente ${ }^{1-3}$.

La brucelosis es una infección endémica en el área mediterránea, originada por una bacteria del género Brucella de la que existen 4 subtipos que afecten al ser humano: B. melitensis, B. suis, $B$. Abortus, B. canis. La B. melitensis es la más patógena. El hombre se contagia por contacto profesional o por la ingesta de productos contaminados como es nuestro caso. La presentación clínica suele ser sistémica en forma de fiebre ondulante (Fiebre de Malta, Fiebre del Mediterráneo y otras) con aparición aguda o crónica. En ocasiones se focaliza en determinados aparatos lo que da lugar a las afecciones localizadas ${ }^{4}$.

La forma localizada más frecuente es la osteoarticular seguida de abscesos esplénicos, infecciones genitourinarias, formas pulmonares $\mathrm{y}$ endocarditis.

La forma osteoarticular representa el 25-40\% de formas localizadas y suele hacerlo como sacroileitis en jóvenes y espondilodiscitis lumbar en edad más avanzada ${ }^{5-7}$. La manifestación clínica más frecuente en estos casos consiste en impotencia funcional, ciatalgia de distribución S1, do- lor abdominal y en ocasiones cuadro abigarrado que puede provocar la realización de una laparotomía exploradora.

En escasos pacientes se produce diseminación con la consecuente formación de abscesos. Ésta puede ser bien vía hematógena: abscesos en hígado, mama, piel; bien por contiguidad: $12 \%$ de los individuos puede complicarse con la formación de abscesos epidurales o paravertebrales ${ }^{8}$, la formación de abscesos de psoas es excepcional $^{7,9,10}$.

El diagnóstico de los abscesos de psoas brucelósicos suele retrasarse por la gran variabilidad clínica y frecuente ausencia de fiebre ${ }^{6,7}$. La mayor parte de pruebas habituales de laboratorio no son útiles, pero existen pruebas serológicas específicas como: sero-aglutinación en tubo para brucella, antibrucella-coombs, aglutinación Rosa de Bengala y ELISA. Los medios de cultivo son lo más específico pero sólo crecen bacterias en un 80\% de los casos: medio de Ruiz Castañeda.

Para el diagnóstico de los AP las pruebas de imagen son fundamentales: la ECO proporciona un instrumento barato, fácil e inocuo, que además del diagnóstico permite el drenaje percutáneo de las colecciones. Presenta el inconveniente de poseer una baja especificidad y sensibilidad $40 \%{ }^{1,3,11,12}$ pues no detecta AP de pequeño tamaño, ni en estado flemonoso, además de presentar las limitaciones generales de la técnica: obesidad, aire interpuesto...

Existe unanimidad para admitir que la (TAC) detecta entre el 90-100\% de los AP, informando de su localización, tamaño y procesos concomitantes. Nos permite el seguimiento así como el tratamiento de las lesiones. Algunos autores mencionan la dificultad para diferenciar tumor, hematoma y absceso ${ }^{1,12}$. La Resonancia Nuclear Magnética (RNM) por su elevado costo y escasa disponibilidad no se hace aconsejable ${ }^{1}$, si bien detecta antes que la TAC las lesiones óseas ${ }^{13,14}$, mostrando una imagen hipointensa en secuencias T1 e hiperintensa en T2.

Para el tratamiento se recomienda como pauta antibiótica de elección: doxiciclina (100mgr/12h) o tetraciclinas 3-6 semanas+estreptomicina $15 \mathrm{mg} /$ $\mathrm{kg} / 12 \mathrm{~h}$ i.m. las 2 primeras semanas, pero por mala penetración de los aminoglucósidos en hueso y su bajo rendimiento en medio ácido y anae- 
robio en ocasiones existen fracasos ${ }^{6,7}$. En estos casos las tetraciclinas + rifampicina 900mgr/día o gentamicina (dosis no definida) parecen ser eficaz. Ante la existencia de absceso si no se procede al drenaje, el tratamiento antibiótico debe mantenerse un minimo de 2 meses.

Cuando existe absceso de gran tamaño se puede llegar a requerir el drenaje percutáneo y en casos seleccionados la cirugía abierta ${ }^{15}$ : destrucciones óseas graves, abscesos extensos, compresión estructuras vecinas, mala respuesta al tratamiento conservador. Se debe individualizar en cada caso.

\section{REFERENCIAS}

1. Laguna P, Moya M. Absceso del músculo psoas: análisis de 11 casos y revisión de la bibliografía. Enferm Infecc Microbiol Clin 1998;16:19-24.

2. Penado S, Espina B, Campo JF. Absceso de psoas. descripción de una serie de 23 casos. Enferm Infecc Microbiol Clin 2001;19:257-260.

3. Conde C, Estébanez J, Rodríguez A. Absceso primario de psoas. A propósito de un caso. Actas Urol Esp 1999;23(4): 374-378.

4. Abad A, Solano D, Ibarnia J. Absceso de psoas: dificil orientación clínica inicial. Enferm Infecc Microbiol Clin 2000;18: 54-55.

5. Dudler J, Balagué F, Waldburguer M. Bilateral primary brucellar abscess. British Journal of Rheumathology 1994; 33:988-990.

6. Baena JM, Alegret F, de Otero J. Absceso de psoas secundario a sacroileitis brucelar. Med Clin 1995;104(9):359.
7. Martín-Davila P, Cobo J, Fortún J. Absceso de psoas secundario a sacroileitis brucelar. Resolución con tratamiento conservador. Enferm Infecc Microbiol Clin 1997; 15: 45-46.

8. Metota Sacristán L, Acha Arrieta V, Gardicia de Lucas MD, et al. Espondilitis y absceso bilateral del psoas por Brucilla: An Med Interna 1995 Sep;12(9):465-466.

9. González-Gay MA, García Porrúa C, Ibañez D. Osteoarticular complications of brucellosis in an Atlantic Area of Spain; J Rheumatol 1999;26:141-145.

10. Aygen B, Esel D, Erdogan N, et al. Psoas Abscess Secondary to Spondylitis. Turk J Med Sci 2002;32:81-83.

11. Tejido A, Jiménez MM, Duarte JM, Villacampa F, Martín MP, Lozano F, Leiva O. Tratamiento percutáneo de los abscesos retroperitoneales. Actas Urol Esp 2000;24(2):131137.

12. García E, Gutiérrez J, Díaz M. Absceso del psoas: presentación de ocho casos y revisión de la literatura. Rev Clin Esp 1995; 195:289-293.

13. Abad A, Solano D, Ibarnia J. Absceso de psoas: difícil orientación clínica inicial. Enferm Infecc Microbiol Clin 2000;18:54-55.

14. Olivares D, Navarro-López V, López García F. Brucelosis complicada con absceso de músculo psoas. Enferm Infecc Microbiol Clin 2004;22(3):197-200.

15. Colmenero JD, Fernández Nebro A, Reguero J.M. et al. Absceso de psoas secundario a sacroileitis brucelar. J Infect 1991;22:107-109.

Dr. J. Romero Otero

Hospital Universitario 12 Octubre

Avda. de Córdoba s/n

28041 Madrid

(Trabajo recibido el 3 noviembre 2004) 